\title{
Avaliação da influência do entorno no uso das estações de bicicletas compartilhadas
}

\author{
Evaluation of environment influence on the use of shared bicycle stations
}

Paola Pol Saraiva[a] (iD), Lauro André Ribeiro[a] , Alcindo Neckel[a] (D), Juliano Lima da Silva[a],

Richard Thomas Lermen[b]

[a] Faculdade Meridional - IMED, Programa de Pós-graduação Strictu Sensu em Arquitetura e Urbanismo, Passo Fundo, RS, Brasil

[b] Faculdade Meridional - IMED, Programa de Pós-graduação Strictu Sensu em Engenharia Civil, Passo Fundo, RS, Brasil

Como citar: Saraiva, P. P., Ribeiro, L. A., Neckel, A., Silva, J. L., \& Lermen, R. T. (2019). Avaliação da influência do entorno no uso das estações de bicicletas compartilhadas. urbe. Revista Brasileira de Gestão Urbana, 11, e20180105. https://doi.org/10.1590/2175-3369.011.001.A013

\section{Resumo}

0 adensamento urbano causa inúmeros problemas de mobilidade, como os congestionamentos, os acidentes de trânsito e a dificuldade de deslocamento para a população. A busca de novas soluções para esses problemas de mobilidade urbana se tornou cada vez mais necessária para proporcionar melhor qualidade de vida à população. Nesse contexto, a Prefeitura Municipal de Passo Fundo, Rio Grande do Sul, recentemente, implementou um sistema de compartilhamento de bicicletas, denominado "Passo Fundo Vai de Bici" para ajudar na solução do problema de mobilidade urbana. Assim, o presente trabalho tem como principal objetivo a análise desse sistema de compartilhamento de bicicletas, a fim de diagnosticar e caracterizar as estações de bicicletas e seus entornos. Por meio dessa análise, foi possível identificar as regiões com maior e menor potencial para viagens de bicicleta. Os resultados demonstraram que as características dos entornos das estações de bicicletas influenciam em sua utilização, com a constatação de que a presença de ciclovia é o fator mais influente quanto ao uso das bicicletas compartilhadas na cidade. Por meio desta pesquisa, tornou-se possível propor duas equações que permitem estimar o potencial de utilização de cada estação. Esses modelos matemáticos podem auxiliar a municipalidade na tomada de decisão quanto à implantação de novas estações.

Palavras-chave: Mobilidade urbana. Mobilidade ativa. Bicicletas compartilhadas. Estações de bicicleta.

\section{Abstract}

Urban densification caused several mobility difficulties such as congestion, traffic accidents and displacement distress for the population. New solutions to urban mobility problems become increasingly necessary to provide better quality of life for users. In this context, The City Hall of Passo Fundo/RS-Brazil

PPS é arquiteta e urbanista, mestre em Arquitetura e Urbanismo, e-mail: paolapol.arquitetura@gmail.com

LAR é professor, doutor em sistemas Sustentáveis de Energia, e-mail: lauro.ribeiro@imed.edu.br

AN é professor, doutor em Geografia e Ciências Ambientais, e-mail: alcindo.neckel@imed.edu.br

JLS é professor, mestre em Arquitetura e Urbanismo, e-mail: juliano_lima_silva@hotmail.com

RTL é professor, doutor em Engenharia de Minas, Metalúrgica e de Materiais, e-mail: richard.lermen@imed.edu.br 
recently implemented a bicycle sharing system, called "Passo Fundo Vai de Bici", in order to assist in the solution of urban mobility difficulties. Therefore, the present work aims to analyze the abovementioned bicycle sharing system in order to diagnose and characterize bicycle stations and their environments. Through this analysis we identified the regions with greatest and least potential for cycling rides. The results showed that the characteristics of the bicycle station environments influence its use, and that the presence of a bicycle path is the most influential factor in the use of shared bicycles in the city. This research led to the proposal of two equations that allow estimating the potential of each station's utilization. These mathematical models can help the municipality in the decision making regarding the implantation of new stations.

Keywords: Urban mobility. Active mobility. Shared bicycles. Bicycle stations.

\section{Introdução}

As crescentes taxas de urbanização ocorridas no Brasil e intensificadas no final do século XX geram inúmeros impactos nas cidades. Adjacente a esse fato, a cultura da valorização do automóvel, motivada, principalmente, pela criação de políticas públicas de incentivo à indústria automobilística, aumenta cada vez mais o número de veículos motorizados (Kunz et al., 2017). Para Minayo (2009), Barczak \& Duarte (2012) e Rubim \& Leitão (2013), as principais consequências consistem no aumento dos congestionamentos, dos acidentes de trânsito, da poluição sonora e atmosférica, das alterações climáticas, bem como na diminuição da qualidade de vida da população, que enfrenta grandes dificuldades de deslocamento diariamente.

O Departamento Nacional de Trânsito registrou um aumento da frota motorizada de 95,58\% nos últimos 10 anos (DENATRAN, 2007a; 2017a). Ademais, de acordo com o último Censo, no ano de 2010 havia cerca de 1 automóvel para cada 2,94 habitantes brasileiros. Por esse motivo, de acordo com Gomide (2006) e Rubim \& Leitão (2013), locomover-se nas cidades torna-se cada vez mais difícil, por causa da falta de infraestrutura e da precariedade dos serviços de transporte coletivo, da cultura de valorização do automóvel ou da dificuldade de efetivar as políticas públicas nacionais e municipais destinadas à mobilidade urbana.

Essas discussões, segundo Ambrosino et al. (2016) e Lowry \& Loh (2017), vêm ganhando espaço à medida que a população sente os impactos referentes a essa dificuldade de deslocamento. No entanto, na busca por soluções para o desafio de mobilidade urbana, surge a mobilidade ativa, a qual, segundo Buehler \& Pucher (2012) e Bandeira et al. (2017), pode ser entendida como aquela que utiliza unicamente o esforço físico do ser humano para locomoção, tornando-se alternativa para mitigação dos problemas de deslocamentos. Para Schuijbroek et al. (2013), o uso da bicicleta e a caminhada são as formas mais comuns de mobilidade ativa e também consideradas as maneiras mais sustentáveis de locomoção.

Entre as alternativas de inserção da mobilidade ativa, para Shaheen et al. (2010) e Chardon et al. (2017), os sistemas de bicicletas compartilhadas vêm ganhando popularidade em inúmeras cidades, em escala mundial. Segundo Demaio (2009) e o Instituto de Políticas de Transporte e Desenvolvimento (IPTD, 2014), os sistemas de bicicletas compartilhadas são um conjunto de estações espalhadas pela malha urbana, contendo bicicletas disponíveis para a população utilizá-las e realizar seus deslocamentos.

A primeira tentativa de compartilhar bicicletas, segundo Demaio (2009), ocorreu em 1965 em Amsterdã, Holanda, com o intuito de reduzir o tráfego de automóveis na cidade. No entanto, apenas em meados de 1995, a cidade de Copenhague, Dinamarca, replicou a ideia e introduziu o sistema de compartilhamento de bicicletas visando a melhorias relacionadas à problemática do furto de bicicletas. Essa fase ficou conhecida como a segunda geração do sistema (Demaio, 2009; IPTD, 2014).

A terceira geração foi implantada em inúmeras cidades da Europa, e um caso de destaque dessa fase foi a cidade de Rennes, na França, que implantou o sistema em 1998, inserindo a tecnologia de cartão inteligente. Segundo o IPTD (2014), Demaio (2009) e Bauman et al. (2017), essa tecnologia possibilitou 
o controle de uso das bicicletas em tempo real, provendo maior segurança, monitoramento e aperfeiçoamento do sistema de cobrança.

A partir dessas experiências exitosas, o interesse por essa forma de deslocamento aumentou também em países fora da Europa. Segundo Demaio (2009), a partir do ano de 2008, surgiram os primeiros casos de implantação de estações de bicicleta compartilhadas em países, como Brasil, Chile, China, Nova Zelândia, Coreia do Sul, Taiwan e Estados Unidos. Ainda, em 2008, havia cerca de 90 sistemas em operação e, em 2009, cerca de 120. De acordo com o IPTD (2014), as últimas inovações são a presença de placas solares nas estações, estações móveis e uso de cartões universais que permitiram a integração entre modais de transporte.

Com base no mapa mundial de bicicletas compartilhadas desenvolvido por Meddin \& Demaio (2018), em maio de 2018 cerca de 1.600 cidades no mundo possuíam o sistema de compartilhamento de bicicletas em operação e cerca de 400 cidades tinham o sistema em fase de construção ou planejamento.

Demaio (2009), Shaheen et al. (2010), Goodman et al. (2014), Ricci (2015), Kabra et al. (2016), Zhang et al. (2017) e Bauman et al. (2017) afirmam que a utilização de sistemas de bicicletas compartilhadas tende a gerar muitos benefícios, tais como: aumento da flexibilidade de mobilidade, por oferecer um transporte alternativo; redução das emissões de poluentes; diminuição dos congestionamentos; aumento da acessibilidade para uma parcela maior da população; resiliência e flexibilidade nas viagens; redução nas barreiras para explorar o ciclismo urbano; aumento da visibilidade das bicicletas; além de uma série de benefícios para a saúde pública.

Nesse contexto, a cidade de Passo Fundo, localizada na região Sul do Brasil, com uma população estimada de 198 mil habitantes (IBGE, 2018) e uma frota de 123.828 veículos motorizados (DENATRAN, 2017b), oferece um cenário ideal para que sejam realizados estudos voltados ao incremento da mobilidade ativa. Isso porque a cidade não foi planejada para comportar esse fluxo de veículos.

O aumento da frota motorizada na cidade de Passo Fundo alcançou 77,87\% nos últimos 10 anos (DENATRAN, 2007b; 2017b), o que torna a bicicleta um transporte alternativo em potencial para melhorar o sistema viário, o qual registra congestionamentos em sua principal via de circulação, a Avenida Brasil, com cerca de $10 \mathrm{~km}$ de extensão.

Nesse sentido, em maio de 2016, a Prefeitura Municipal de Passo Fundo inaugurou um sistema de compartilhamento de bicicletas denominado "Passo Fundo Vai de Bici", o qual conta com 10 estações e um total de 110 bicicletas e 150 vagas para depositá-las. Os moradores que desejarem utilizar o sistema devem realizar um cadastro para que possam usufruir diária e gratuitamente do serviço.

Desse modo, o principal objetivo do presente trabalho foi levantar e analisar dados quantitativos e qualitativos do sistema de compartilhamento de bicicletas em seu segundo ano de operação na cidade de Passo Fundo/RS. Assim, realizou-se uma estimativa quanto ao potencial de utilização de cada estação de bicicleta compartilhada e o entendimento das características do entorno, bem como o desenvolvimento de um modelo matemático que possibilita estimar quais são os melhores locais para implantar novas estações.

Sendo assim, este estudo mostra a importância de criar e analisar modelos cicloviários que venham a favorecer a mobilidade ativa, já que, como apontado anteriormente, grandes e médias cidades estão enfrentando graves problemas relacionados à locomoção dos seus moradores. Ainda, pesquisas que envolvam o uso da bicicleta como transporte podem contribuir para possíveis projetos de melhoria para a mobilidade urbana.

\section{Metodologia}

A metodologia utilizada parte da análise dos entornos de cada estação de bicicleta e da identificação dos possíveis pontos ou características geradoras de demanda para o sistema, tais como: praças, parques, instituições de ensino, ciclovias, entre outros. Com base no reconhecimento das características dos entornos de cada estação, variáveis (critérios) quantitativas foram definidas. Esses critérios foram 
relacionados com o histórico de dados reais de utilização das estações, gerando um modelo matemático, o qual possibilitou estimar o potencial de uso das estações, isto é, a quantidade de bicicletas retiradas e/ou devolvidas em função dos critérios estabelecidos.

Zhang et al. (2017) afirmam que o impacto das particularidades urbanas de diferentes regiões da cidade influenciam diretamente nas escolhas da população local, pois, quanto maior a densidade, a diversidade, a acessibilidade e a infraestrutura dedicada às bicicletas, maior será a aceitação por parte dos usuários. Nesse sentido, os procedimentos metodológicos foram organizados em quatro etapas:

Etapa I: para descrever a atual situação do sistema "Passo Fundo Vai de Bici", levando-se em consideração que ele está em seu segundo ano de operação, a coleta de dados foi realizada com a Prefeitura Municipal de Passo Fundo, na qual relatórios mensais referentes à utilização do sistema foram analisados. Tais dados referem-se ao perfil dos usuários do sistema, isto é, ao número de viagens realizadas em cada estação, aos horários e aos dias em que havia maior utilização das bicicletas, bem como aos dados gerais, como número total de viagens realizadas e número total de quilômetros percorridos. Esses dados dizem respeito ao intervalo do dia 11 de maio de 2016, quando se iniciou a operação do sistema na cidade, até o dia 15 de maio de 2018, totalizando, aproximadamente 24 meses de operação.

Ademais, por meio desses relatórios, foi coletado o histórico de utilização de cada estação, ou seja, o número de bicicletas retiradas e devolvidas em cada uma das estações, entre os meses de abril de 2017 e abril de 2018, totalizando 12 meses.

Etapa II: realizou-se o levantamento da atual situação das 10 estações da cidade e seus entornos, já que autores, como Vogel et al. (2011), afirmam que o uso de cada estação depende de seus arredores. Assim, para esse levantamento, foi considerada a área de abrangência de cada estação varrida por um raio de, aproximadamente, 500 metros (IPTD, 2014), isso como forma de identificar os principais pontos e características que contribuem para a geração de viagens.

Nessa etapa, a ferramenta Google Maps foi utilizada para mapear e identificar a presença de pontos de interesse, tais como: escolas, faculdades, praças, centros comerciais, entre outros. A Figura 1 representa o mapa com a localização de cada estação na malha da cidade de Passo Fundo.

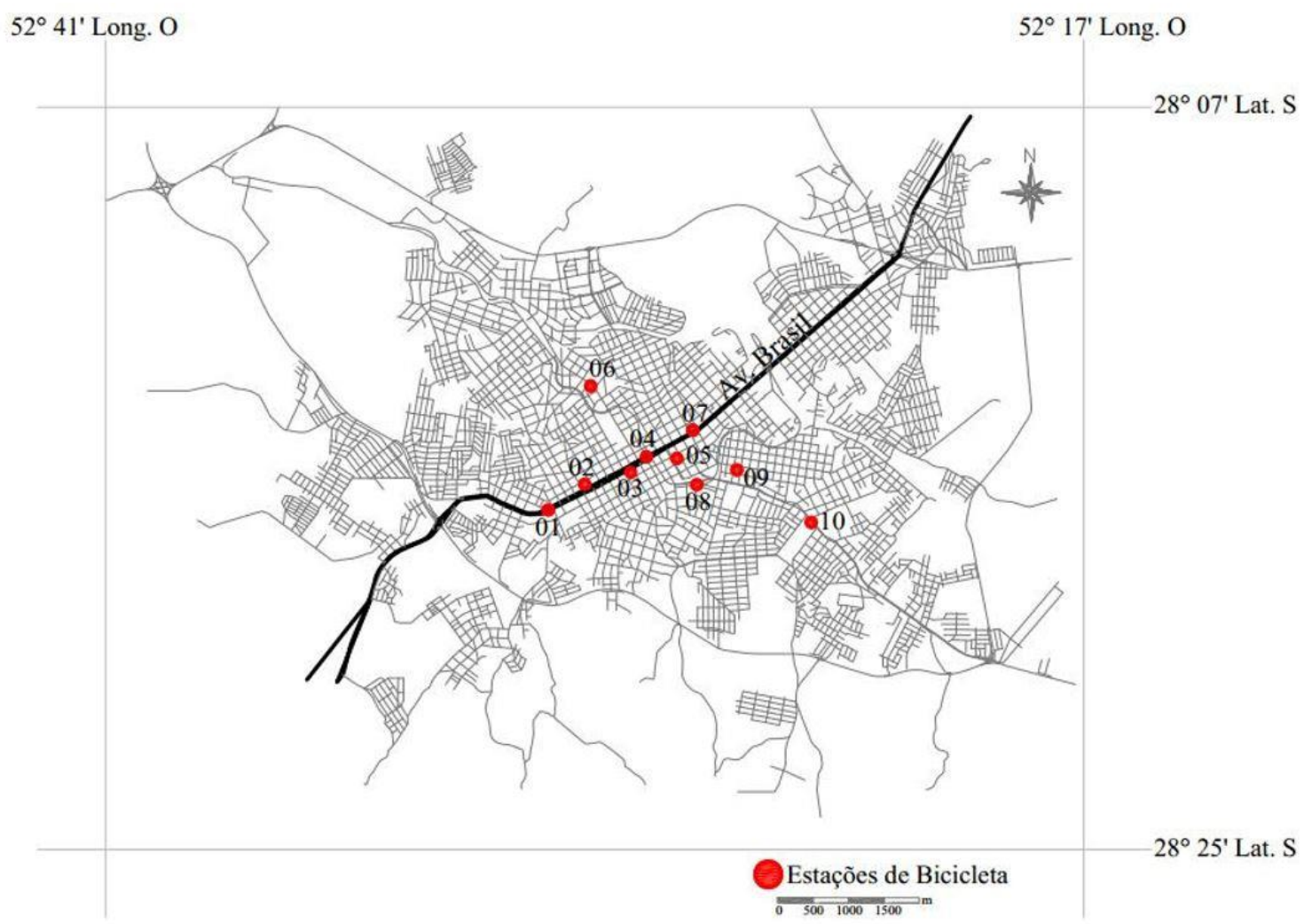

Figura 1 - Localização das 10 estações na cidade de Passo Fundo. Fonte: elaborada pelos autores (2018). 
Etapa III: realizou-se uma análise dos entornos das 10 estações levantadas anteriormente a partir de cinco critérios, os quais são variáveis contínuas, pois tratam-se de características mensuráveis que podem assumir valores em uma escala contínua.

Esses critérios, que também foram utilizados nas pesquisas do IPTD (2014), Saraiva et al. (2017), Chardon et al. (2017) e Zhang et al. (2017), são:

- Educação (E): quantidade de instituições de ensino no entorno (escolas de ensino fundamental e médio, faculdades e universidades).

- Áreas verdes (V): medida da área verde dentro da área de abrangência de cada estação, isto é, foram consideradas as áreas de parques, praças e canteiros no entorno de cada estação.

- Segurança no tráfego (ST): distância, em metros, entre a estação e as ciclovias ou ciclofaixas mais próximas para realização da viagem.

- Distância da estação mais próxima (DE): menor distância, em metros, entre duas estações.

- População potencial (PP): população em potencial na área de abrangência de cada uma das estações calculada de acordo com a densidade populacional em cada uma delas.

Esse último critério (população potencial) utilizado na presente avaliação dos entornos relaciona-se à densidade populacional, destacada pelo IPTD (2014) como fundamental para planejar e implementar um sistema de compartilhamento de bicicletas em uma cidade. Para esse critério, utilizaram-se os dados coletados com a Prefeitura Municipal de Passo Fundo quanto à densidade populacional de cada setor urbano da cidade no qual se encontram as estações de bicicleta. Após, calculou-se a área de abrangência de cada estação (raio de 500 metros) e multiplicou-se pela população presente por $\mathrm{km}^{2}$ em cada setor em que existem estações instaladas. Os dados são mostrados na Tabela 1.

Tabela 1 - Dados utilizados para cálculo da população abrangida em cada estação, sendo a área de abrangência $0,785 \mathrm{~km}^{2}$ para cada uma delas

\begin{tabular}{ccc}
\hline Estação & Densidade (habitantes/(km²) & População potencial \\
\hline 1. Boqueirão Legal & 5373 & 4219 \\
2. IE & 5373 & 4219 \\
3. Notre Dame & 7878 & 6187 \\
4. Teixeirinha & 7878 & 6187 \\
5. Marechal Floriano & 7878 & 6187 \\
6. Vera Cruz & 5017 & 3940 \\
7. Praça Tochetto & 7878 & 6187 \\
8. Parque da Gare & 7878 & 6187 \\
9. Praça Santa Terezinha & 5521 & 4336 \\
10. Brigada Militar & 4197 & 3296 \\
\hline
\end{tabular}

Fonte: elaborada pelos autores (2018).

Segundo Rybarczyk \& Wu (2010), além dos critérios citados anteriormente, a topografia da cidade também influencia a utilização de bicicletas. No entanto, ao analisar os entornos das estações do "Passo Fundo Vai de Bici", constatou-se que elas foram instaladas em áreas com pouca variação de altitude. Por esse motivo, optou-se por desconsiderar a variável de topografia.

Etapa IV: posteriormente, utilizando um software de planilha eletrônica, a partir do histórico de informações quantitativas de bicicletas (retiradas e devoluções mensais) levantadas na primeira etapa da pesquisa e das variáveis criadas a partir dos critérios citados, foram realizadas análises empíricas de dados por meio de regressão linear do histórico de uso de bicicletas das 10 estações. Consequentemente, foram obtidas duas equações de primeira ordem aditiva, uma para as bicicletas retiradas e outra para as bicicletas devolvidas, considerando a influência dos critérios previamente estabelecidos.

Buscou-se, por meio das equações, estimar quantitativamente o uso (retiradas e devoluções) de bicicletas em estações por meio das variáveis que caracterizam seus entornos. Foram considerados válidos os critérios com um nível de significância (P-valor) de 0,05 (5\%) ou menos. 


\section{Resultados e discussões}

\section{O "Passo Fundo Vai de Bici" no $24^{\circ}$ mês de operação}

Os dados coletados por meio de relatório de controle mensal com a Prefeitura Municipal mostraram que, até maio de $2018,68,2 \%$ dos usuários das bicicletas compartilhadas eram do sexo masculino. Quanto à faixa etária, 43,5\% dos usuários possuíam entre 20 e 30 anos, e 25,6\%, de 30 a 40 anos, mostrando que o sistema atingia, principalmente, a população de 20 a 40 anos da cidade. Quanto à escolaridade, observou-se que a maior parcela de usuários (29\%) possuía ensino médio completo, 25,9\%, ensino superior incompleto, e 13,5\%, ensino superior completo. Quanto à renda, 35,4\% dos usuários declararam que não possuíam nenhuma fonte de renda, 18,1\%, até 1 salário mínimo, e 24,9\%, entre 1 e 2 salários mínimos, indicando que, em geral, os usuários possuíam baixos rendimentos financeiros.

Quanto ao uso, 76\% dos usuários declararam que utilizavam as bicicletas durante os dias da semana. Quanto aos horários, 23\% usavam-nas pela parte da manhã (das $6 \mathrm{~h}$ às 11h59), 47\%, pela parte da tarde (das $12 \mathrm{~h}$ às $17 \mathrm{~h} 59$ ), e $30 \%$, pela parte da noite (das $18 \mathrm{~h}$ às $22 \mathrm{~h}$ ).

Ainda segundo os dados gerais fornecidas pela Prefeitura Municipal de Passo Fundo, o número de cadastrados no sistema até maio de 2018 era de 26.002 usuários, ultrapassando 10\% da população total da cidade de Passo Fundo.

O total de quilômetros percorridos era de $1.998 .614 \mathrm{~km}$, calculado de acordo com a distância entre o ponto de retirada da bicicleta até o ponto de sua devolução. Em caso de retiradas e devoluções na mesma estação, calculava-se essa distância estimando uma velocidade média de $12 \mathrm{~km} / \mathrm{h}$. Além disso, a média de viagens diárias foi de 219.

A área total de cobertura do sistema foi de $5,67 \mathrm{~km}^{2}$, considerando que algumas estações possuíam área de cobertura sobrepostas. A densidade das estações (estações por $\mathrm{km}^{2}$ na área de cobertura) foi de 1,76.

A Figura 2 apresenta a demanda por estação, de acordo com o número de bicicletas retiradas e devolvidas até o dia 15 de maio de 2018. Pode-se visualizar que 4 das 5 estações localizadas na Avenida Brasil (01 a 04), principal via da cidade, que concentra importantes atividades de comércio e de serviços e apresenta maior segurança para circulação de bicicletas devido à existência de trechos de ciclovias e ciclofaixas, foram as mais utilizadas.

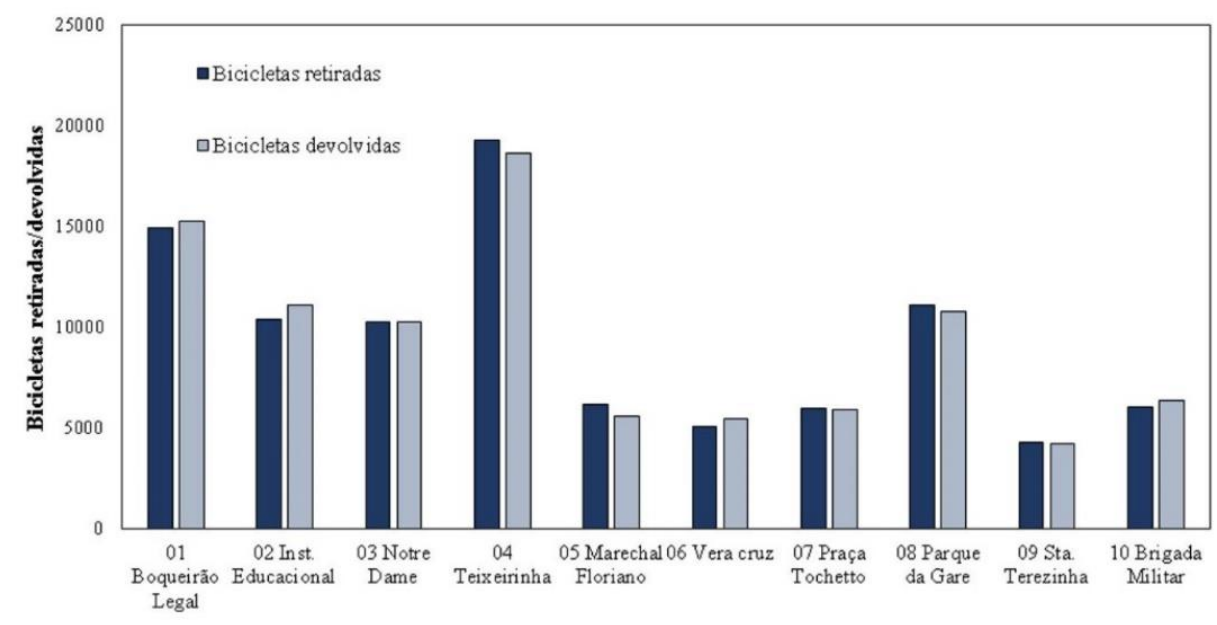

Figura 2 - Demanda por estação de acordo com o número de bicicletas retiradas e devolvidas nos 24 meses de operação do sistema. Fonte: elaborada pelos autores (2018).

A estação Parque da Gare (08) também foi uma das mais utilizadas devido ao fato de o parque ter sido revitalizado recentemente, atraindo inúmeras pessoas diariamente para lazer ou prática de esportes. Nota-se também que as estações mais distantes do centro da cidade, como Brigada Militar (10), Praça Santa Terezinha (09) e Vera Cruz (06), foram as menos utilizadas, com exceção das estações Praça 
Marechal Floriano (05) e Praça Tochetto (07), que localizam-se em área central mas estavam entre as menos utilizadas.

Nas Figuras 3 e 4, é possível visualizar o número de bicicletas retiradas e devolvidas em cada estação entre os meses de março de 2017 e abril de 2018. Nota-se que há variação quanto ao número de bicicletas retiradas e devolvidas durante os meses analisados. Nesse sentido, destaca-se que a cidade de Passo Fundo possui estações climáticas bem definidas, com inverno cujas temperaturas chegam próximo de $0^{\circ} \mathrm{C}$. Desse modo, quanto ao uso das bicicletas compartilhadas, de forma geral, nota-se que, nos meses em que as temperaturas eram mais baixas na cidade, ou seja, de abril a agosto, o número de usos foi reduzido, enquanto, nos meses entre setembro até fevereiro, quando as temperaturas eram mais elevadas, o uso foi mais intenso em todas as estações de bicicleta.

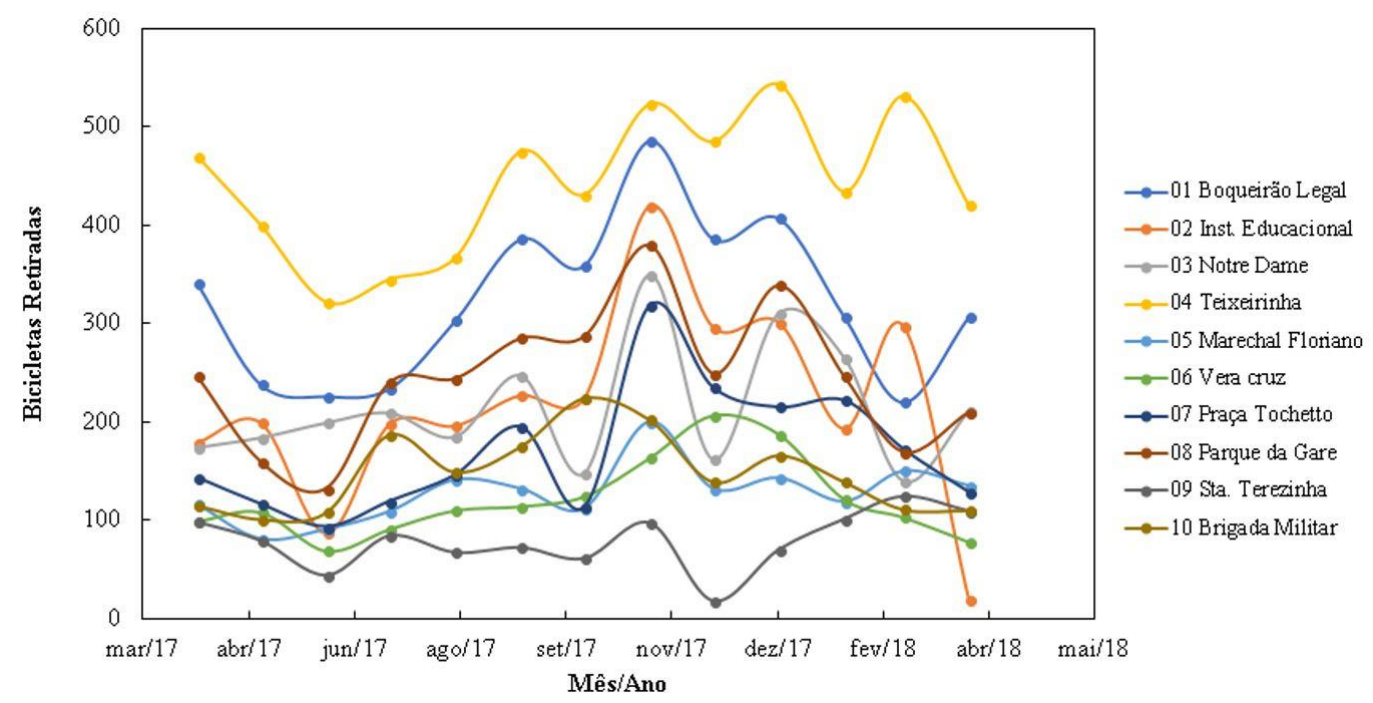

Figura 3 - Quantidade de bicicletas retiradas em função do período de coleta de dados para cada estação. Fonte: elaborada pelos autores (2018).

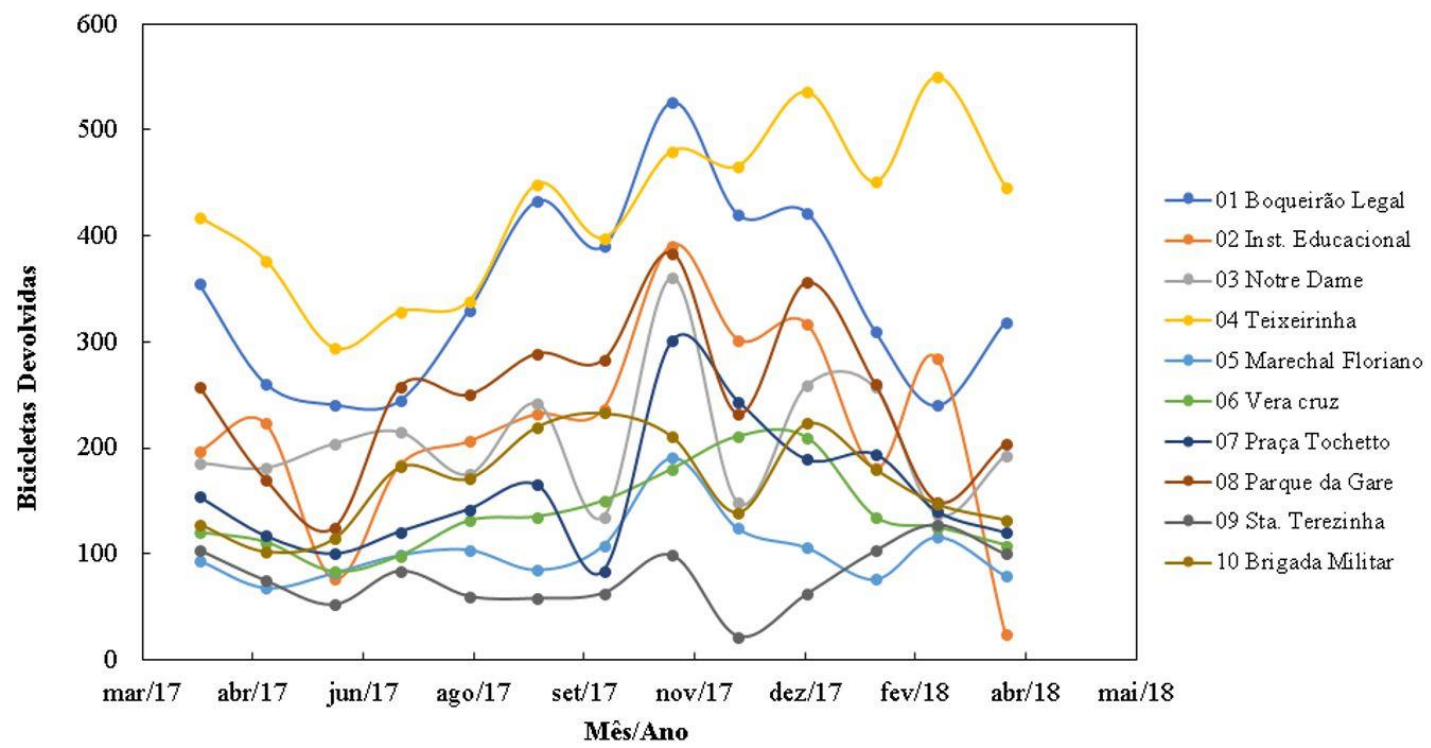

Figura 4 - Quantidade de bicicletas devolvidas em função do período de coleta de dados para cada estação. Fonte: elaborada pelos autores (2018).

Com esses dados gerais, torna-se possível analisar o funcionamento do sistema e, a partir dessa análise, elencar algumas recomendações encontradas na literatura acerca dos sistemas de compartilhamento de bicicletas. Primeiramente, conforme Fishman et al. (2013) e o IPTD (2014), um 
bom indicador de desempenho é o número de viagens por dia por bicicleta, que deve ser de 4 a 8 . Segundo os dados coletados, o sistema analisado realizou cerca de 2,19 viagens por bicicleta por dia. Além disso, o IPTD (2014) recomenda que haja de 10 a 16 estações por km², ou seja, cerca de 1 estação a cada 300 m. Em Passo Fundo, as estações estavam posicionadas com uma distância média de $680 \mathrm{~m}$.

\section{Reconhecimento dos entornos de cada estação}

Nesta etapa, analisou-se o entorno de cada estação (raio de aproximadamente $500 \mathrm{~m}$ do entorno de cada uma delas) (IPTD, 2014), a fim de identificar os principais possíveis pontos geradores de demanda de viagens, ou seja, locais onde ocorrem importantes atividades diariamente, potencializando o uso das estações existentes (Tabela 2).

Tabela 2 - Levantamento dos possíveis pontos gerados de demanda em cada estação

\begin{tabular}{|c|c|}
\hline Estação & Possíveis pontos geradores de demanda \\
\hline 1. Boqueirão Legal & $\begin{array}{c}\text { Canteiro central (utilizado como praça) } \\
\text { Instituto Educacional Metodista } \\
\text { Faculdade Fasurgs } \\
\text { Instituto Menino Deus } \\
\text { Escola Municipal Notre Dame }\end{array}$ \\
\hline 2. Inst. Educacional & $\begin{array}{l}\text { Canteiro central (utilizado como praça) } \\
\text { Colégio Joaquim Fagundes dos Reis } \\
\text { Instituto Educacional Metodista } \\
\text { Praça da Mãe Preta }\end{array}$ \\
\hline 3. Notre Dame & $\begin{array}{c}\text { Canteiro central (utilizado como praça) } \\
\text { Colégio Notre Dame } \\
\text { Escola Círculo Operário } \\
\text { Escola Nicolau Araújo Vergueiro } \\
\text { Praça Tamandaré } \\
\text { Praça da Mãe Preta } \\
\text { Hospital São Vicente de Paula } \\
\text { Faculdade de Medicina UPF } \\
\text { Universidade Federal da Fronteira Sul } \\
\text { Shopping Bella Cittá } \\
\text { Área de intenso comércio }\end{array}$ \\
\hline 4. Teixeirinha & $\begin{array}{c}\text { Shopping Bella Cittá } \\
\text { Praça Marechal Floriano } \\
\text { Praça Tamandaré } \\
\text { Faculdade Anhanguera } \\
\text { Colégio Notre Dame } \\
\text { Escola Círculo Operário } \\
\text { Escola Nicolau Araújo Vergueiro } \\
\text { Hospital São Vicente de Paula } \\
\text { Faculdade de Medicina UPF } \\
\text { Universidade Federal da Fronteira Sul } \\
\text { Área de intenso comércio }\end{array}$ \\
\hline 5. Marechal Floriano & $\begin{array}{c}\text { Praça Marechal Floriano } \\
\text { Praça Tochetto } \\
\text { Escola Protásio Alves } \\
\text { Faculdade Anhanguera } \\
\text { Shopping Bella Cittá } \\
\text { Parque da Gare (parte) } \\
\text { Área de intenso comércio }\end{array}$ \\
\hline 6. Vera Cruz & $\begin{array}{c}\text { Hospital Ortopédico e da Visão } \\
\text { Parque Banhado da Vergueiro } \\
\text { Escola João de Césaro } \\
\text { Serviço Nacional de Aprendizagem Industrial (SENAI) } \\
\text { Escola Ernesto Tochetto } \\
\text { Área de comércio moderado }\end{array}$ \\
\hline
\end{tabular}


Tabela 2 - Continuação...

\begin{tabular}{cc}
\hline Estação & Possíveis pontos geradores de demanda \\
\hline Praça Tochetto \\
Escola Protásio Alves \\
7. Praça Tochetto & Folégio Marista Conceição \\
& Clube Recreativo Juvenil \\
Hospital da Cidade & Hospital Pronto Clínica \\
& Praça Antônio Xavier \\
Área de intenso comércio \\
8. Parque da Gare & Parque da Gare \\
& Faculdade de Tecnologia Senac \\
& Praça Marechal Floriano \\
& Área de intenso comércio \\
\hline 9. Staça Capitão Jovino \\
Colégio Menino Jesus \\
Instituto Meridional (IMED) e IFIBE \\
Colégio Tiradentes \\
Escola Monte Castelo \\
Parque da Gare (parte) \\
\hline 10. Brigada Militar & Instituto Estadual Cecy Leite Costa \\
& Praça do Cecy \\
& Escola Georgina Rosado \\
\hline
\end{tabular}

Fonte: elaborada pelos autores (2018).

Segundo García-Palomares et al. (2012) e Ho \& Szeto (2017), um dos principais fatores que influenciam o sucesso de implantação das bicicletas compartilhadas é a localização das estações, já que elas devem estar posicionadas de acordo com a demanda potencial de usuários, ou seja, próximas aos locais onde ocorrem atividades importantes e conectadas a outras modalidades de transporte, a fim de maximizar a área de cobertura de cada uma delas.

Recomenda-se que a implantação do sistema inicialmente concentre as estações em zonas centrais, onde há maior densidade, e gradualmente se expanda até as áreas mais periféricas, como os bairros e as áreas industriais, para que funcione como um sistema integrado de transporte (IPTD, 2014). Além disso, García-Palomares et al. (2012) e Bauman et al. (2017) enfatizam que a capacidade de cada estação (número de bicicletas e vagas livres) deve ser calculada de acordo com a demanda de cada região.

De acordo com o IPTD (2014) e Bauman et al. (2017), a área de cobertura de cada estação corresponde a um raio de 500 metros do entorno dela e deve conter um significativo número de pontos de origem e de destino. Portanto, ao analisar o mapa de abrangência das estações e considerando que várias delas possuíam área de cobertura em comum, a área de cobertura total do sistema foi de $5,68 \mathrm{~km}^{2}$.

Além disso, os levantamentos realizados demostraram que 7 das 10 estações estavam localizadas próximas a escolas, 9 das 10 estações localizavam-se em praças e parques ou muito próximas a estes, 7 estações situavam-se próximas a instituições de ensino superior e somente 1 estação localizava-se perto de uma indústria.

Pode-se observar que metade das estações estavam localizadas na Avenida Brasil, principal via da cidade, que conecta inúmeras áreas importantes. Somente as estações Vera Cruz (06) e Brigada Militar (10) foram implantadas em bairros afastados da área central da cidade.

Frade \& Ribeiro (2014) afirmam que os sistemas de compartilhamento de bicicletas podem ser utilizados como um serviço isolado ou como um serviço intermodal, complementado com outras modalidades de transportes. No caso do "Passo Fundo Vai de Bici", as bicicletas do sistema eram utilizadas, na maioria dos casos, para complementar viagens, pelo fato de a maioria dos bairros da cidade não possuir estações. 


\section{Análise dos entornos de cada estação}

A Tabela 3 mostra os dados levantados em cada uma das 10 estações de acordo com os cinco critérios elencados.

Tabela 3 - Avaliação dos cinco critérios nas 10 estações

\begin{tabular}{cccccc}
\hline Estações & $\begin{array}{c}\text { Educação- } \\
\mathbf{E}\end{array}$ & $\begin{array}{c}\text { Áreas verdes } \\
\mathbf{V}\left(\mathbf{m}^{2}\right)\end{array}$ & $\begin{array}{c}\text { Segurança no } \\
\text { tráfego - ST }(\mathbf{m})\end{array}$ & $\begin{array}{c}\text { Distância da estação mais } \\
\text { próxima - DE }(\mathbf{m})\end{array}$ & $\begin{array}{c}\text { População } \\
\text { potencial - PP }\end{array}$ \\
\hline 01 & 4 & 21890 & 0 & 690 & 4219 \\
02 & 2 & 45719 & 0 & 655 & 4219 \\
03 & 6 & 57627 & 0 & 300 & 6187 \\
04 & 6 & 41373 & 0 & 300 & 6187 \\
05 & 2 & 37000 & 523 & 530 & 6187 \\
06 & 3 & 15638 & 1270 & 1390 & 3940 \\
07 & 3 & 42560 & 790 & 780 & 6187 \\
08 & 1 & 79396 & 0 & 711 & 6187 \\
09 & 4 & 36050 & 520 & 711 & 4336 \\
10 & 2 & 8172 & 1550 & 1350 & 3296 \\
\hline
\end{tabular}

Fonte: elaborada pelos autores (2018).

Por fim, realizaram-se duas análises de regressão linear multivariada, relacionando os critérios escolhidos para a criação das variáveis analisadas nas 10 estações com os dados reais de utilização das bicicletas em cada estação, sendo uma regressão linear para as bicicletas retiradas e uma para as bicicletas devolvidas, no período de 12 meses analisados. Foi utilizada a Equação (1) para regressão.

$Y=\beta_{0}+\sum_{i=1}^{n} \beta_{i}^{*} X_{i}$

Em que: $Y$ é a variável dependente; $\beta_{0}$, a constante; $\beta_{i}$, o coeficiente de regressão; e $X_{i}$, as variáveis independentes. Para as regressões, a constante $\beta_{0}$ foi considerada 0 , porém, para garantir o funcionamento adequado da equação nessa situação, devem ser respeitadas as seguintes condições de intervalo das variáveis: PP > 0, devido ao fato de que se necessita de uma população potencial mínima para haver retiradas de bicicletas; $\mathrm{DE}>0$, pois deve considerar a estação mais próxima, levando em conta que haja uma distância a ser percorrida. A Equação 2 apresenta a regressão linear para as bicicletas retiradas.

$Y_{R}=13,4079 * E-0,0037 * V-0,2598 * S T+0,2183 * D E+0,0524 * P P$

Em que: $Y_{R}$ é a quantidade de retiradas de bicicletas; $E$ (Educação), $V$ (Áreas verdes), $S T$ (Segurança no tráfego), $D E$ (Distância da estação mais próxima) e $P P$ (População potencial), os critérios que foram descritos anteriormente.

A regressão linear considerando o número de bicicletas devolvidas e os cinco critérios elencados na pesquisa é apresentada por meio da Equação 3.

$Y_{D}=15,7222 * E-0,0036 * V-0,2830 * S T+0,2692 * D E+0,0453 * P P$

Em que: $Y_{D}$ corresponde ao número de bicicletas devolvidas nas estações.

0 coeficiente de determinação ( $\left.\mathrm{R}^{2}\right)$ da Equação 2 foi de 86,51\%, e da Equação 3, de 85,93\%. De acordo com o P-valor, todas as variáveis tiveram influência significativa com confiabilidade de $95 \%$ ou mais, 
conforme pode ser visto nas Tabelas 4 e 5, que mostram as análises de variância para os modelos matemáticos de bicicletas retiradas e devolvidas.

Tabela 4 - Análise de variância para a quantidade de bicicletas retiradas

\begin{tabular}{ccccccc}
\hline Critérios & $\begin{array}{c}\text { Grau de } \\
\text { liberdade }\end{array}$ & $\begin{array}{c}\text { Soma dos } \\
\text { quadrados }\end{array}$ & $\begin{array}{c}\text { Soma dos quadrados } \\
\text { ajustada }\end{array}$ & $\begin{array}{c}\text { Média dos quadrados } \\
\text { ajustada }\end{array}$ & F-valor & P-valor \\
\hline E & 1 & 5637427 & 42460 & 42460 & 5,238 & 0,0238 \\
V & 1 & 345489 & 140836 & 140836 & 17,374 & 0,0000 \\
ST & 1 & 9581 & 464225 & 464225 & 57,269 & 0,0000 \\
DE & 1 & 290839 & 307802 & 307802 & 37,972 & 0,0000 \\
PP & 1 & 214382 & 214382 & 214382 & 26,447 & 0,0000 \\
Erro & 125 & 1013263 & 1013263 & 8106 & & \\
\hline
\end{tabular}

Fonte: elaborada pelos autores (2018).

Tabela 5 - Análise de variância para a quantidade de bicicletas devolvidas

\begin{tabular}{ccccccc}
\hline Critérios & $\begin{array}{c}\text { Grau de } \\
\text { liberdade }\end{array}$ & $\begin{array}{c}\text { Soma dos } \\
\text { quadrados }\end{array}$ & $\begin{array}{c}\text { Soma dos quadrados } \\
\text { ajustada }\end{array}$ & $\begin{array}{c}\text { Média dos quadrados } \\
\text { ajustada }\end{array}$ & F-valor & P-valor \\
\hline E & 1 & 5574579 & 58132 & 58132 & 6,849 & 0,0099 \\
V & 1 & 298660 & 137893 & 137893 & 16,247 & 0,0000 \\
ST & 1 & 1612 & 550179 & 550179 & 64,825 & 0,0000 \\
DE & 1 & 449672 & 467707 & 467707 & 55,107 & 0,0000 \\
PP & 1 & 160958 & 160958 & 160958 & 18,965 & 0,0000 \\
Erro & 125 & 1060898 & 1060898 & 8487 & & \\
\hline
\end{tabular}

Fonte: elaborada pelos autores (2018).

Também, por meio das análises de variância, pode-se afirmar que o critério de maior influência sobre as retiradas e as devoluções de bicicletas foi a segurança no tráfego (ST), pois, estatisticamente, quanto maior o valor de F, mais influente é o fator. Esse resultado corrobora pesquisa realizada pela Companhia de Engenharia de Tráfego (CET, 2014), a qual mostrou que, após a implantação de um trecho de ciclovia em uma importante avenida da cidade de São Paulo (Brigadeiro Faria Lima), o número de ciclistas em circulação aumentou $37 \%$ nessa via. Isso também confirma a importância da presença de infraestrutura dedicada à bicicleta, já que garante maior segurança e conforto para os usuários.

Ao analisar as Equações 2 e 3, verificou-se que duas das cinco variáveis consideradas, $V$ e $S E$, obtiveram sinal negativo. Quanto ao sinal negativo da variável $S E$, entende-se que, quanto mais distante uma estação se encontra de um trecho cicloviário, menos bicicletas serão utilizadas. Constatou-se que, de forma geral, esse foi o comportamento das estações do "Passo Fundo Vai de Bici", já que estações posicionadas ao longo dos trechos cicloviários existentes eram as mais utilizadas.

0 sinal negativo da variável $V$ (áreas verdes) ocorre, possivelmente, porque as estações localizadas na Praça Marechal Floriano e na Praça Tochetto, as quais possuem abundantes áreas verdes em seus entornos, estavam entre as estações menos utilizadas, ou seja, o fato de as estações estarem posicionadas próximas a áreas verdes em Passo Fundo/RS não garante que elas sejam intensamente utilizadas.

Essa constatação corrobora a necessidade de uma amostragem maior para que a equação possa ajustar-se e para que possam ser alcançados resultados mais precisos, já que somente foram consideradas as 10 estações existentes baseando-se nos cinco critérios elencados e desconsiderando outras variáveis não medidas e que podem influenciar no uso das estações.

As Equações 2 e 3 serviram para avaliação do sistema de variáveis, ou seja, dos cinco critérios analisados e avaliados nos entornos, podendo ser verificado na Figura 5, que mostra a comparação entre os valores coletados com a Prefeitura Municipal (média das bicicletas retiradas e devolvidas) e os valores previstos pelo modelo matemático. 


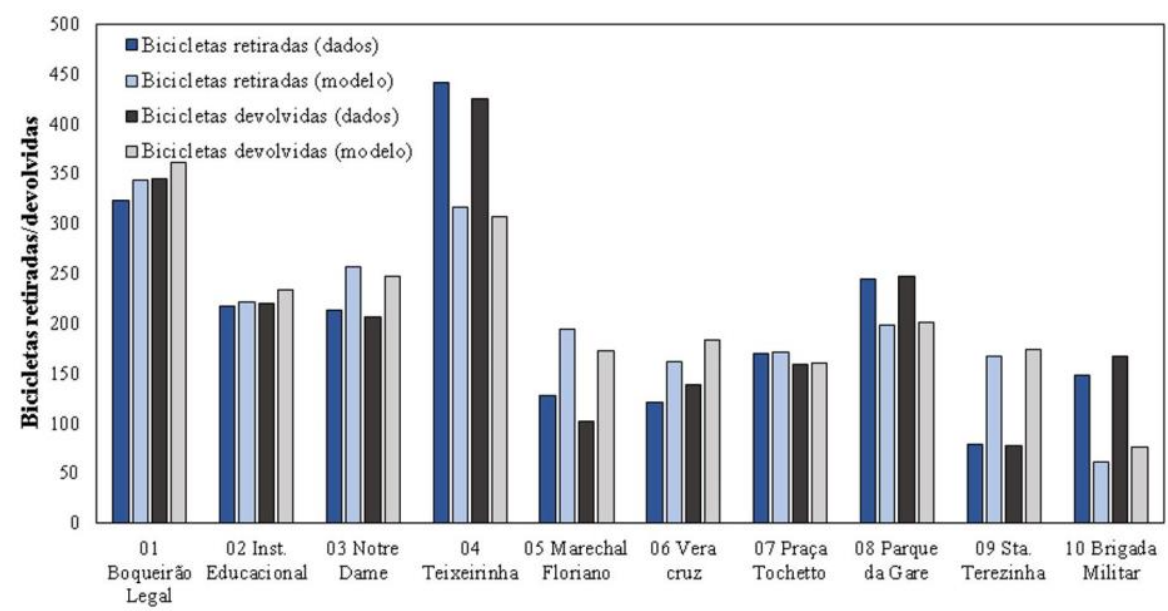

Figura 5 - Média de bicicletas retiradas e devolvidas nas diferentes estações por mês. Fonte: elaborada pelos autores (2018).

Observa-se que, de forma geral, os dados seguiram um mesmo comportamento. Contudo, pode-se observar que, nas estações Brigada Militar, Marechal Floriano, Santa Terezinha e Teixeirinha, houve maiores dispersões entre os dados reais e os dados estimados. Acredita-se que esses resultados ocorreram devido ao fato de existirem outras variáveis não consideradas neste estudo por dificuldades de medição e que possuem influência no uso dessas estações, como a presença de áreas de intenso comércio e prestação de serviços.

Portanto, sugere-se que, em estudos futuros, sejam realizadas novas análises quanto a outros critérios que possam contribuir para o uso das bicicletas compartilhadas como forma de alcançar resultados mais precisos e que sejam consideradas as áreas de intenso comércio, como a proximidade com shopping center, bares e restaurantes, entre outros locais que geram atratividade diária.

Porém, pode-se afirmar que os resultados apresentados permitiram compreender o funcionamento do sistema "Passo Fundo Vai de Bici", além de demonstrar como o entorno das estações de bicicletas e as características da cidade afetam diretamente a utilização do sistema. Foi possível compreender os aspectos mais influentes, como a presença de ciclovias e a proximidade entre as estações, que possibilitam a criação de redes voltadas à ciclomobilidade, facilitando os deslocamentos ativos, e os aspectos menos influentes, como a presença de instituições de ensino, de áreas verdes e a população potencial.

Acredita-se que a presença de instituições de ensino tenha sido pouco significativa por causa de a Prefeitura Municipal de Passo Fundo ter estabelecido uma regra de que somente maiores de 18 anos poderiam utilizar o sistema, o que significa que boa parte dos alunos das instituições de ensino fundamental e médio não tinha acesso às bicicletas compartilhadas.

Quanto à variável população potencial, acredita-se que ela esteja entre as menos influentes, já que, segundo a literatura, as estações de bicicleta podem ser dimensionadas de acordo com a população em potencial da área de cobertura de cada uma delas (IPTD, 2014).

Com base nos resultados alcançados, espera-se que este estudo possa contribuir para possíveis projetos de expansão da rede na cidade, avaliando-se os critérios mais influentes que podem potencializar o uso das bicicletas compartilhadas.

Além disso, essse sistema de avaliação de critérios pesquisados pode ser replicado em outras áreas, utilizando-se da equação para contribuir para o processo avaliativo do potencial de utilização de bicicletas em novas regiões. Vogel et al. (2011), Lin \& Yang (2011), García-Palomares et al. (2012), Martinez et al. (2012), IPTD (2014) e Tran et al. (2015) confirmam a necessidade de estudos voltados ao entendimento das variáveis que influenciam na utilização das bicicletas compartilhadas. Esses estudos tendem a colaborar com a tomada de decisão quanto à localização das estações e podem evitar implantações pouco úteis que tendem a gerar custos desnecessários. 


\section{Conclusões}

Com o objetivo de realizar uma análise quantitativa e qualitativa do sistema "Passo Fundo Vai de Bici" e analisar o potencial de utilização de cada estação de acordo com as características dos entornos, este trabalho demonstrou que a avaliação dos diferentes cenários e dos cinco critérios em cada uma das estações tornou possível visualizar a demanda de cada uma delas e as estações com maior potencial.

As estações Boqueirão Legal (01), Teixeirinha (04) e Parque da Gare (08) foram as estações mais utilizadas e com grande potencial de acordo com as características de seu entorno. Recomenda-se então a ampliação da capacidade dessas estações quando o sistema for expandido.

Também foi possível identificar as estações menos utilizadas, como as estações Vera Cruz (06) e Sta. Terezinha (09), que, apesar de possuírem pontos geradores de viagens no entorno, estavam localizadas afastadas da área central, distantes das outras estações, e, principalmente, não possuíam ciclovias contínuas em seus entornos, variável mais significativa para gerar deslocamentos de acordo com esta análise.

Contudo, destaca-se que essas estações possuem papel importante para a dinâmica do sistema, pois permitem que o sistema abranja áreas mais periféricas da cidade e alcancem uma parcela maior de usuários, possibilitando que estes partam de suas residências para os locais de interesse. Ademais, sugere-se que, a partir de novos estudos, sejam criados novos trechos de ciclovia na cidade como forma de potencializar a utilização dessas estações e promover maior segurança e conforto para os ciclistas passo-fundenses.

Ainda, a partir da criação das equações, embora se tenha detectado a necessidade de melhorias, tornase possível avaliar o potencial de diferentes locais para implantação de novas estações de bicicleta, de acordo com as características destacadas neste estudo. Elas podem colaborar com processos decisórios de futuros projetos de extensão do programa "Passo Fundo Vai de Bici", a fim de evitar implementação de estações pouco utilizadas, as quais podem gerar custos desnecessários para o poder público local. Além disso, essa metodologia pode ser adaptada para outras cidades, desde que os critérios a serem avaliados sejam revisados e ajustados à realidade de cada local.

\section{Referências}

Ambrosino, G., Nelson, D. J., Boero, M., \& Pettinelli, I. (2016). Enabling intermodal urban transport through complementary services: from flexible mobility services to the shared use mobility agency. Transportation Economics, 59, 179-184. http://dx.doi.org/10.1016/j.retrec.2016.07.015.

Bandeira, A. S., Silva, K. S., Del Duca, G. F., Zilch, G. R., Oliveira, E. S. A., Barroa, M. G. V., \& Nahas, M. V. (2017). Factors associated with bicycle use for commuting and for leisure among Brazilian workers. Sport Sciences for Health, 13(1), 1-6.

Barczak, R., \& Duarte, F. (2012). Impactos ambientais da mobilidade urbana: cinco categorias de medidas mitigadoras. Urbe. Revista Brasileira de Gestão Urbana, 4(1), 13-32. http://dx.doi.org/10.1590/S217533692012000100002.

Bauman, A., Crane, M., Drayton, B. A., \& Titze, S. (2017). The unrealised potential of bike share schemes to influence population physical activity levels - A narrative review. Preventive Medicine, 103S, 1-17. PMid:28223189.

Buehler, R., \& Pucher, J. (2012). Walking and cycling in Western Europe and United States. Recuperado em 18 de agosto de 2017, de http://onlinepubs.trb.org/onlinepubs/trnews/trnews280WesternEurope.pdf

Companhia de Engenharia de Tráfego - CET. (2014). Ciclovias em SP: integrando e fazendo o bem para a cidade. Recuperado em 20 de fevereiro de 2018, de http://www.cetsp.com.br/media/316505/sp\%20400km_v2s.pdf

Chardon, C. M., Caruso, G., \& Thomas, I. (2017). Bicycle sharing system 'success' determinants. Transportation Research Part A, Policy and Practice, 100(1), 202-214. http://dx.doi.org/10.1016/j.tra.2017.04.020. 
Demaio, P. (2009). Bike-sharing: History, Impacts, Models of Provision, and Future. Journal of Public Transportation, 12(4), 41-56. http://dx.doi.org/10.5038/2375-0901.12.4.3.

Departamento Nacional de Trânsito - DENATRAN. (2007a). Frota de veículos no Brasil por ano. Recuperado em: 20 de maio de 2018, de http://www.denatran.gov.br/index.php/estatistica/237-frota-veiculos

Departamento Nacional de Trânsito - DENATRAN. (2007b). Frota de veículos no Brasil por município. Recuperado em: 20 de maio de 2018, de http://www.denatran.gov.br/index.php/estatistica/237-frota-veiculos

Departamento Nacional de Trânsito - DENATRAN. (2017a). Frota de veículos no Brasil por ano. Recuperado em: 20 de maio de 2018, de http://www.denatran.gov.br/index.php/estatistica/237-frota-veiculos

Departamento Nacional de Trânsito - DENATRAN. (2017b). Frota de veículos no Brasil por município. Recuperado em: 20 de maio de 2018, de http://www.denatran.gov.br/index.php/estatistica/237-frota-veiculos

Fishman, E., Washington, S., \& Haworth, N. (2013). Bike share: a synthesis of the literature. Transport Reviews, 33(2), 148-165. http://dx.doi.org/10.1080/01441647.2013.775612.

Frade, I., \& Ribeiro, A. (2014). Bicycle sharing systems demand. Procedia: Social and Behavioral Sciences, 111, 518527. http://dx.doi.org/10.1016/j.sbspro.2014.01.085.

García-Palomares, J. C., Gutiérrez, J., \& Latorre, M. (2012). Optimizing the location of stations in bike-sharing programs: a GIS approach. Applied Geography (Sevenoaks, England), 35(1-2), 235-246.

http://dx.doi.org/10.1016/j.apgeog.2012.07.002.

Gomide, A. A. (2006). Mobilidade urbana, iniquidade e políticas sociais. In IPEA. Políticas sociais acompanhamento e análise (pp. 242-250). Brasília: IPEA.

Goodman, A., Green, J., \& Woodcock, J. (2014). The role of bicycle sharing systems in normalising the image of cycling: an observational study of London cyclists. Journal of Transport \& Health, 1(1), 5-8.

http://dx.doi.org/10.1016/j.jth.2013.07.001. PMid:25568838.

Ho, S. C., \& Szeto, W. Y. (2017). A hybrid large neighborhood search for the static multi-vehicle bike-repositioning problem. Transportation Research Part B: Methodological, 95, 340-363. http://dx.doi.org/10.1016/j.trb.2016.11.003.

Instituto Brasileiro de Geografia e Estatística - IBGE. (2018). Censo 2010. Recuperado em 15 de maio de 2018, de www.ibge.gov.br/cidadesat

Instituto de Políticas de Transporte e Desenvolvimento - IPTD. (2014) Guia de planejamento de sistemas de bicicletas compartilhadas. Recuperado em 10 de outubro de 2017, de https://www.itdp.org/wpcontent/uploads/2013/12/ITDP-Brasil_Guia-de-Planejamento-de-Sistemas-de-Bicicletas-Compartilhadas.pdf

Kabra, A., Belavina, E., \& Girotra, K. (2016). Bike-share systems: accessibility and availability. Recuperado em 22 de fevereiro de 2017, de https://ssrn.com/abstract=2555671

Kunz, M., Neckel, A., \& Kujawa, H. A. (2017). The influence of public policies on urban mobility: a comparative study between Porto Alegre (Brazil) and Washington D.C. (United States). Journal of Civil Engineering and Architecture, 11(3), 295-304.

Lin, J., \& Yang, T. (2011). Strategic design of public bicycle sharing systems with service level constraints. Transportation Research Part E, Logistics and Transportation Review, 47(2), 284-294.

http://dx.doi.org/10.1016/j.tre.2010.09.004.

Lowry, M., \& Loh, T. H. (2017). Quantifying bicycle network connectivity. Preventive Medicine, 95(Suppl), $134-140$. http://dx.doi.org/10.1016/j.ypmed.2016.12.007. PMid:27939970.

Martinez, L. M., Caetano, L., Eiró, T., \& Cruz, F. (2012). An Optimisation Algorithm to Establish the Location of Stations of a Mixed Fleet Biking System: An Application to the City of Lisbon. Procedia: Social and Behavioral Sciences, 54, 513-524. http://dx.doi.org/10.1016/j.sbspro.2012.09.769.

Meddin, R., \& Demaio, P. (2018). The bike share world map. Recuperado em 25 de maio de 2018, em http://www.metrobike.net/the-bike-sharing-world-map/ 
Minayo, M. C. S. (2009). Seis características das mortes violentas no Brasil. Revista Brasileira de Estudos de Populacao, 26(1), 135-140. http://dx.doi.org/10.1590/S0102-30982009000100010.

Ricci, M. (2015). Bike sharing: a review of evidence on impacts and processes of implementation and operation. Research in Transportation Business \& Management, 15, 28-38. http://dx.doi.org/10.1016/j.rtbm.2015.03.003.

Rubim, B., \& Leitão, S. (2013). O Plano de Mobilidade Urbana e o futuro das cidades. Estudos Avançados, 27(79), 5566. http://dx.doi.org/10.1590/S0103-40142013000300005.

Rybarczyk, G., \& Wu, C. (2010). Bicycle facility planning using GIS and multi-criteria decision analysis. Applied Geography (Sevenoaks, England), 30(2), 282-293. http://dx.doi.org/10.1016/j.apgeog.2009.08.005.

Saraiva, P. P., Ribeiro, L. A., Neckel, A., Lermen, R. T., \& Silva, J. L. (2017). Evaluating the bicycle sharing system in a medium-sized city. International Journal of Humanities and Social Science, 7(5), 65-74.

Schuijbroek, J., Hampshire, R. C., \& Van Hoeve, W. J. (2013). Inventory rebalancing and vehicle routing in bike sharing systems. European Journal of Operational Research, 257(3), 992-1004.

http://dx.doi.org/10.1016/j.ejor.2016.08.029.

Shaheen, S., Guzman, S., \& Zhang, H. (2010). Bikesharing in Europe, the Americas, and Asia. Transportation Research Record: Journal of the Transportation Research Board, 2143(1), 159-167. http://dx.doi.org/10.3141/2143-20.

Tran, T. D., Ovtracht, N., \& D’arcier, B. F. (2015). Modeling Bike Sharing System using Built Environment Factors. Procedia Cirp, 30, 293-298. http://dx.doi.org/10.1016/j.procir.2015.02.156.

Vogel, P., Greiser, T., \& Mattfeld, D. C. (2011). Understanding bike-sharing systems using data mining: exploring activity patterns. Procedia: Social and Behavioral Sciences, 20, 514-523.

http://dx.doi.org/10.1016/j.sbspro.2011.08.058.

Zhang, Y., Thomas, T., Brussel, M., \& Maarseveen, M. (2017). Exploring the impact of built environment factors on the use of public bikes at bike stations: case study in Zhongshan, China. Journal of Transport Geography, 58, 59-70. http://dx.doi.org/10.1016/j.jtrangeo.2016.11.014.

Editor: Fábio Duarte

Recebido: Jun. 11, 2018

Aprovado: Jul. 09, 2018 